\title{
Stereocorrelation Formalism Considering Brightness and Contrast Effects: Application to Torsional Loadings
}

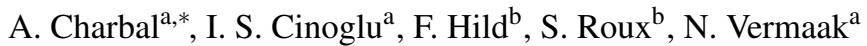 \\ ${ }^{a}$ Department of Mechanical Engineering \& Mechanics, Lehigh University, Bethlehem, PA 18015, USA \\ ${ }^{b}$ Laboratoire de Mécanique et Technologie (LMT), ENS Paris-Saclay, CNRS, Université Paris-Saclay, \\ 94235 Cachan Cedex, France
}

\begin{abstract}
Many multiaxial experimental setups rely on stereocorrelation (SC) techniques to resolve surface strains and deformations. Torsional loadings can affect the quality and accuracy of SC results due to changes in lighting conditions and the non-conservation of gray levels with the angle of twist. In this note, the gray level correction method implemented for 2D DIC cases is introduced in a 3D surface NURBS SC formalism and applied for torsional loadings. In particular, tension-torsion loading of AISI 1144 steel rods is used to demonstrate the approach. Different surface parameterizations, with and without gray level corrections, are tested in order to enhance the SC quality (defined via gray level residuals). It is found that both gray level corrections and relaxing the regularization of an overly constrained NURBS surface definition (6 vs. 36 knots), lower SC residuals.

Keywords: StereoCorrelation, Gray level corrections, NURBS surface, Registration residuals, Multiaxial testing
\end{abstract}

The examination of the spatial and temporal changes of SC residuals prior to exploiting the resolved surface strains and deformations is necessary. The residual fields highlight unexpected events in image registration (e.g., an error in the estimation of displacements, a discrepancy in the gray level conservation assumption, the presence

\footnotetext{
${ }^{*}$ Corresponding author

Email addresses: alc517@lehigh.edu (A. Charbal), isc214@lehigh.edu (I. S. Cinoglu), francois.hild@ens-paris-saclay.fr (F. Hild), stephane.roux@ens-paris-saclay.fr (S. Roux), vermaak@lehigh.edu (N. Vermaak)
} 
5 of discontinuities or even indicate a convergence issue in the analysis). A deeper understanding of the residuals provides additional information and guides the user to better parameterize and/or adapt the SC scheme. In this note, it is proposed to analyze the SC residuals for multiaxial loading histories in the limit of small strains/displacements.

There are two main reasons why DIC residuals increase with applied torque. The first one is the change in brightness and contrast due to the angle of twist. Consequently, the underlying assumption of gray level conservation, which lies at the basis of global SC formalisms [2,3] is violated. This issue can be addressed by adding corrections directly in the global SC formalism. The second reason has to do with the parameterization surface that describes the Region of Interest (ROI) on the specimens to be studied. The parameterization is characterized by the degree of the mixing functions, the number of control points and knots [4]. In the following, several cases exploring the influence of modifying these parameters are discussed and some guidelines to adapt the global SC formalism for torsional loadings are presented.

\section{Equipment, Materials, Methods}

Cylindrical rods made of high strength AISI 1144 medium carbon steel with tapered gage section (12.7 $\mathrm{mm}$ in diameter gage) [5] were subjected to quasi-static tensiontorsion loadings using an Axial/Torsional 319.25 MTS testing machine. The tests consisted of linearly ramping up the force from 0 to $94 \mathrm{kN}$. This level was then held constant while the torque was ramped up from 0 to $90 \mathrm{Nm}$. After a dwell of $1 \mathrm{~min}$ at the set force and torque, an additional cyclic axial force was added with an amplitude of $2 \mathrm{kN}$.

A custom-made stereovision system was used to analyze the 3D surface displacement and 2D strain fields on the visible portion of the cylindrical samples during all of the performed tests. The lighting conditions were constant and stable throughout the experiments using a single central lamp. The system consisted of two Proscilica GX3300 GigE cameras, which have definitions of $3296 \times 2472$ pixels. CCD progressive sensors allowed for a maximum frame rate of $17.1 \mathrm{fps}$ at full definition. C-Mount Schneider-Kreuznach 2.8/50 lenses were used on both cameras to reach a resolution of $25 \mu \mathrm{m} /$ pixel. A custom GUI interface was developed, via Matlab, to synchronize both 
cameras and trigger the acquisition with the MTS machine. After each experiment, the images were analyzed using NURBS-based SC [2, 3] for a region of interest (ROI) centered about the rod axis (i.e., approximately 320 pixels across $12.7 \mathrm{~mm}$ ). In the following, the reference images are defined as the first ones captured before any loading is applied to the sample.

40 In order to perform SC, it is necessary to determine the projection matrices relating the $3 \mathrm{D}$ space where the sample is positioned to the $2 \mathrm{D}$ images captured by the camera sensors (Figure 1). In this work, the projection matrices are optimized using the pseudo-kinematic basis within an integrated DIC algorithm [2].


Figure 1: Left and right camera images of the calibration target.

The NURBS [4] representation of the surface is defined in the parametric space $(u, v) \in[0,1]^{2}$ (bold typeface denote vectors in the following)

$$
\boldsymbol{X}(u, v)=\frac{\sum_{i=0}^{m} \sum_{j=0}^{n} N_{i, p}(u) N_{j, q}(v) \omega_{i j} \boldsymbol{P}_{i j}}{\sum_{i=0}^{m} \sum_{j=0}^{n} N_{i, p}(u) N_{j, q}(v) \omega_{i j}}
$$

with

$$
\forall u \in[0,1], N_{i, 0}=\left\{\begin{array}{ccc}
1 & \text { when } & u_{i} \leq u \leq u_{i+1} \\
0 & \text { otherwise } &
\end{array}\right\}
$$

and $u_{i}$ being the components of the control point vector $\left\{u_{0}, \ldots, u_{i}, \ldots, u_{m}\right\}$ considering 
$u_{i} \leq u_{i+1}$, and

$$
N_{i, p}(u)=\frac{u-u_{i}}{u_{i+p}-u_{i}} N_{i, p-1}(u)+\frac{u_{i+p+1}-u}{u_{i+p+1}-u_{i+1}} N_{i+1, p-1}(u),
$$

where $N_{i, p}$ and $N_{j, q}$ are the mixing functions for the two components $u$ and $v$ of the parametric space [4]. The number of control points is $n_{p}=(m+1)(n+1)$ (in the present case $\left.n_{p}=6\right)$. The pair $(p, q)$ represents the degree attributed to the functions describing the surface. $P_{i j}=\left(X_{P_{i j}}, Y_{P_{i j}}, Z_{P_{i j}}\right)$ contain the coordinates of the control points. $\omega_{i j}$ correspond to the weight applied to each knot (for knots at corners the weight is equal to 1 while the knots in the center are equal to 0.83 ; this is necessary to describe a cylindrical surface [4]). The representation of the NURBS surface for the present ROI is shown in Figure 2.
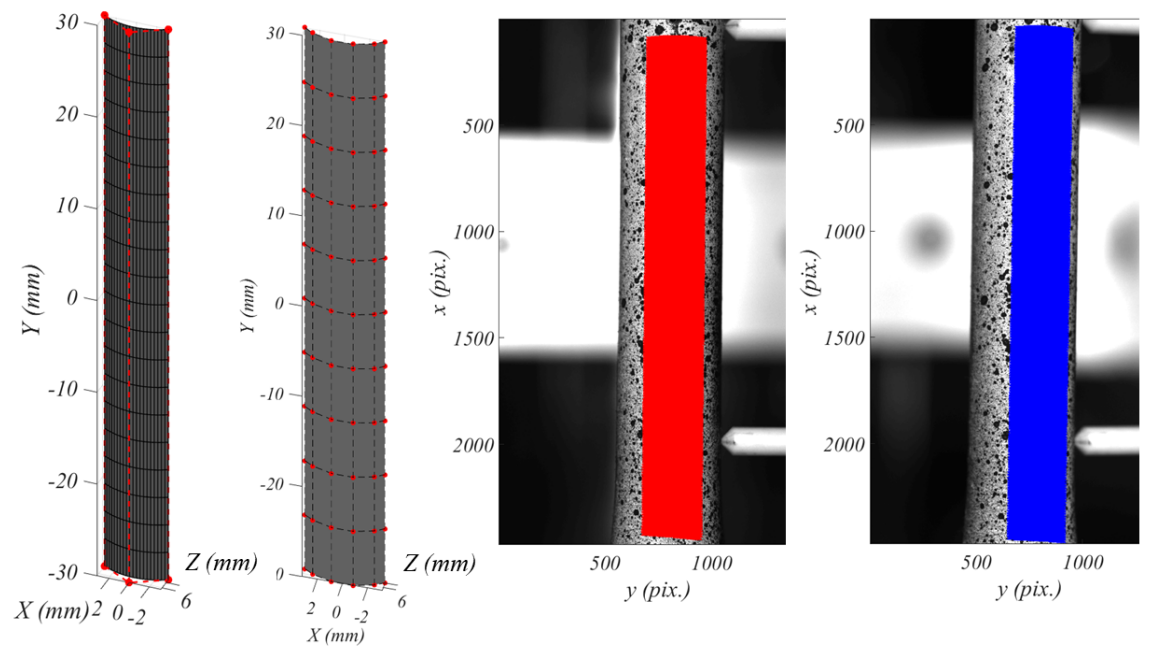

Figure 2: ROI defined by the 3D NURBS surface with 6 and 36 knots. The parameterized surface is also shown when projected on the left (red) and right (blue) camera frame.

\section{Brightness and contrast corrections within global SC}

Torsion-related changes in brightness and contrast (e.g., caused by inhomogeneous lighting) are an issue because they violate the underlying assumption of gray level conservation. Brightness and contrast corrections (BCC) are introduced based on polynomial shape functions [1]. The modified functional to minimize, now considering 
gray level relaxation, becomes

$$
\Gamma_{G L C}=\sum_{R O I}\left(\sum_{i} a_{i}^{r}+\sum_{j}\left(\left(1+b_{j}^{r}\right) f_{0}^{r}\right)-g^{r}\right)^{2}+\sum_{R O I}\left(\sum_{i} a_{i}^{l}+\sum_{j}\left(\left(1+b_{j}^{l}\right) f_{0}^{l}\right)-g^{l}\right)^{2}
$$

where $f^{r, l}$ and $g^{r, l}$ are the reference and deformed images for the left (superscript $l$ ) and right (superscript $r$ ) cameras, $a^{r, l}$ and $b^{r, l}$ brightness and contrast correction fields , respectively. $f_{0}^{r, l}$ represent the initial reference images, before any BCC has been applied. The corrections are applied to reference images. The following simplified notations were used

$$
\begin{gathered}
f^{l, r}=f\left(\boldsymbol{x}^{l, r}\left(u, v, \boldsymbol{P}_{i j}\right)\right), \quad g^{l, r}=g\left(\boldsymbol{x}^{l, r}\left(u, v, \boldsymbol{P}_{i j}+\boldsymbol{d} \boldsymbol{P}_{i j}\right)\right), \\
a^{l, r}=a\left(\boldsymbol{x}^{l, r}\left(u, v, \boldsymbol{P}_{i j}\right)\right), \quad b^{l, r}=b\left(\boldsymbol{x}^{l, r}\left(u, v, \boldsymbol{P}_{i j}\right)\right),
\end{gathered}
$$

where $\boldsymbol{P}_{\boldsymbol{i} j}$ are the initial positions of the control points of the NURBS surface. The incremental brightness and contrast corrections $\delta a_{i}$ and $\delta b_{j}$ are iteratively updated with the incremental displacements, $\boldsymbol{d} \boldsymbol{P}_{\boldsymbol{i} \boldsymbol{j}}$, using an enhanced Newton-Raphson procedure

$$
\left[C_{a b}\right]\{d p\}=\{\boldsymbol{\eta}\}
$$

where $\left[\boldsymbol{C}_{\boldsymbol{a} \boldsymbol{b}}\right]$ is the $\left(3 n_{p}+2 n_{a}+2 n_{b}\right) \times\left(3 n_{p}+2 n_{a}+2 n_{b}\right)$ Hessian $\left(n_{a}\right.$ brightness fields and $n_{b}$ contrast fields)

$$
\left[\boldsymbol{C}_{\boldsymbol{a b}}\right]=\sum^{R O I}\left\{\boldsymbol{s}_{a b}^{r}\right\}\left\{\boldsymbol{s}_{a b}^{r}\right\}^{\top}+\left\{\boldsymbol{s}_{a b}^{l}\right\}\left\{\boldsymbol{s}_{a b}^{l}\right\}^{\top},
$$

and $\left\{\boldsymbol{s}_{a b}^{r, l}\right\}$ the column vector containing the $\left(3 n_{p}+2 n_{a}+2 n_{b}\right)$ scalar products of the sensitivity fields

$$
\begin{aligned}
& \left\{\boldsymbol{s}_{a b}^{r}\right\}=\left\{\left\{\boldsymbol{s}^{r}\right\},\left\{\boldsymbol{\alpha}^{r}\right\},\left\{\boldsymbol{\beta}^{r}\right\},\{\mathbf{0}\},\{\mathbf{0}\}\right\} \\
& \left\{\boldsymbol{s}_{a b}^{l}\right\}=\left\{\left\{\boldsymbol{s}^{l}\right\},\{\mathbf{0}\},\{\mathbf{0}\},\left\{\boldsymbol{\alpha}^{l}\right\},\left\{\boldsymbol{\beta}^{l}\right\}\right\}
\end{aligned}
$$

in which $\left\{\boldsymbol{s}^{r, l}\left(\boldsymbol{x}^{r, l}\right)\right\}$ is the column vector containing the $3 n_{p}$ scalar products of the pixel/3D space sensitivity vector with the corresponding gradient of each reference 
image $\nabla f^{r, l}\left(\right.$ i.e., $\left.\frac{\delta x^{r, l}}{\delta P_{i j}^{r, l}} \cdot \nabla f^{r, l}\right)$ for each point defined in the parametric space $\boldsymbol{x}^{r, l}(u, v)$. The brightness corrections are gathered in the vector $\left\{\boldsymbol{\alpha}^{r, l}\right\}$

$$
\left\{\boldsymbol{\alpha}^{r, l}\left(\boldsymbol{x}^{r, l}\right)\right\}=\left\{\phi_{1}\left(\boldsymbol{x}^{r, l}\right), \ldots, \phi_{j}\left(\boldsymbol{x}^{r, l}\right), \ldots, \phi_{N}\left(\boldsymbol{x}^{r, l}\right)\right\}
$$

and the contrast corrections in vector $\left\{\boldsymbol{\beta}^{r, l}\right\}$

$$
\left\{\boldsymbol{\beta}^{r, l}\left(\boldsymbol{x}^{r, l}\right)\right\}=\left\{\phi_{1} f_{0}^{r, l}\left(\boldsymbol{x}^{r, l}\right), \ldots, \phi_{j} f_{0}^{r, l}\left(\boldsymbol{x}^{r, l}\right), \ldots, \phi_{N} f_{0}^{r, l}\left(\boldsymbol{x}^{r, l}\right)\right\}
$$

The polynomial fields for BCCs are listed in Table 1. In this study the BCC fields are defined by the same shape and number of functions (thus $n_{a}=n_{b}=N$ ).

Table 1: Polynomials for the BCC fields.

\begin{tabular}{|l|l|r|}
\hline Designation & $\mathrm{N}$ & Interpolation fields \\
\hline Constant & 1 & $\phi_{1}=1$ \\
\hline Linear & 3 & $\phi_{2}=u, \phi_{3}=v$ \\
\hline Bilinear & 4 & $\phi_{4}=u v$ \\
\hline Order 2 & 6 & $\phi_{5}=u^{2}, \phi_{6}=v^{2}$ \\
\hline
\end{tabular}

The right hand side member of the linearized S-DIC problem reads

$$
\{\boldsymbol{\eta}\}=\sum_{R O I}\left(\left\{\boldsymbol{s}_{a b}^{r}\right\}\left(f_{n}^{r}-\tilde{g}^{r}\right)+\left\{\boldsymbol{s}_{a b}^{l}\right\}\left(f_{n}^{l}-\tilde{g}^{l}\right)\right),
$$

for the current iteration, where the images $\tilde{g}^{r, l}$ are the deformed images corrected by the surface deformation $\boldsymbol{P}_{\boldsymbol{i} \boldsymbol{j}}+\boldsymbol{d} \boldsymbol{P}_{\boldsymbol{i} \boldsymbol{j}}$, while the reference image is updated according to the BCCs at each iteration $n$

$$
f_{n}^{l, r}=\sum_{i} a_{i}^{l, r}+\sum_{j}\left(1+b_{j}^{l, r} f_{0}^{l, r}\right)
$$

\section{Mitigation Strategies for Torsion-Induced Measurement Errors in SC}

Prior to investigating multiaxial tests, a set of simple rigid body motions was applied to evaluate the performance of the SC code. The measured displacement was $0.083 \mathrm{~mm}$ while $0.080 \mathrm{~mm}$ was applied. Similarly, the observed rotation was 0.94 
while 1.0 degree was applied. At convergence the residual levels were less than $2.0 \%$ body translation and rotation respectively.

In order to assess the effect of BCCs and the degrees of freedom defining the NURBS surface, the following cases were explored and compared with the baseline results (Case \#1):

Case \#1: The number of knots defining the ROI is $n_{p}=6$, and the degree of the mixing functions describing the NURBS surface is 1 .

Case \#2: Same as Case \#1 with BCCs.

Case \#3: The number of knots is $n_{p}=6$, and the degree of the mixing functions is raised to 2 .

Case \#4: Same as Case \#3 with BCCs.

Case \#5: The number of knots defining the ROI is increased to $n_{p}=36$. The degree of the mixing functions is 1 . This provides a finer surface "discretization." Case \#6: Same as Case \#5 with additional BCCs.

\section{Results \& Discussion}

For the multiaxial tests, Figure 3 shows the change of the SC residuals

$$
\rho_{D I C}=\frac{1}{2 \Omega} \cdot \sum_{R O I}\left(\frac{\left|f_{n}^{r}-\tilde{g}^{r}\right|}{\max \left(f^{r}\right)-\min \left(f^{r}\right)}+\frac{\left|f_{n}^{l}-\tilde{g}^{l}\right|}{\max \left(f^{l}\right)-\min \left(f^{l}\right)}\right) \cdot 100,
$$
\#1-6, Table 2 summarizes the SC residuals. 


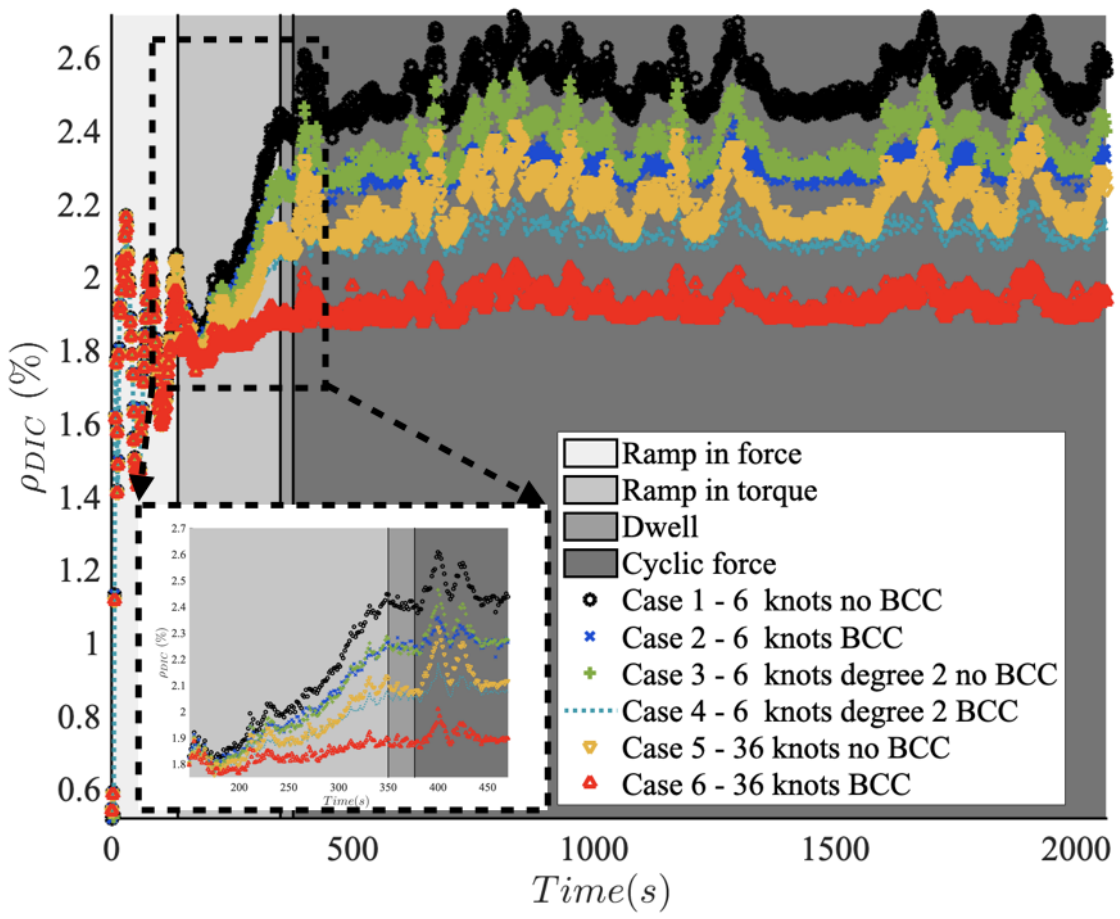

Figure 3: Change of gray level residuals with respect to the load history for different NURBS parameterizations and/or with BCCs.

Table 2: Comparison of residuals for Cases \#1-6.

\begin{tabular}{|c|c|c|c|c|}
\hline Case \# & \# Knots & Degree & BCC & SC Residual (\%) \\
\hline 1 & 6 & 1 & No & 2.5 \\
\hline 2 & 6 & 1 & Yes & 2.3 \\
\hline 3 & 6 & 2 & No & 2.4 \\
\hline 4 & 6 & 2 & Yes & 2.1 \\
\hline 5 & 36 & 1 & No & 2.2 \\
\hline 6 & 36 & 1 & Yes & 1.8 \\
\hline
\end{tabular}

For Case \#1, the residuals fluctuate when the force is linearly ramped up to $90 \mathrm{kN}$. 
However they remain below $2.2 \%$ of the dynamic range of the reference pictures. This result indicates that there is no correlation between the residual levels and the applied force. Conversely, there is a linear increase in the residual levels while applying the torque ramp, thereby suggesting a correlation.

Figure 4 shows gray level residual maps for the left and right cameras at convergence of the images acquired at the end of the force ramp and also at the end of the torque ramp. The results after the force ramp show mostly acquisition noise and interpolation errors. The results after the torque ramp highlight that the residual fields increase non-uniformly in space, which is another indication of a correlation between brightness and contrast variations and angle of twist.

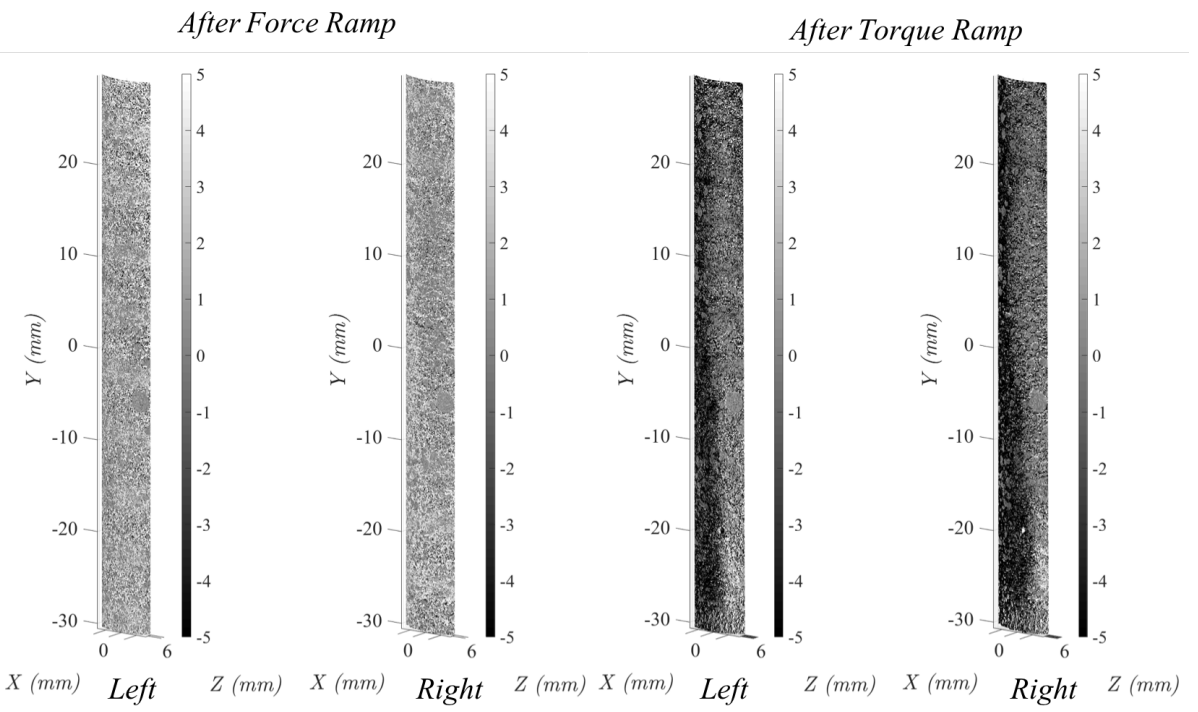

Figure 4: Gray level residual maps for the left and right cameras after applying the force ramp and the torque ramp.

For Case \#2 (where BCCs are applied), the overall residuals decrease from $2.5 \%$ to $2.3 \%$. The BCCs reduce the residuals from $2.4 \%$ to $2.1 \%$ when the degree of the 90 NURBS surface is raised to 2 (comparing Cases \#3 and \#4). The best results are obtained when the BCCs are combined with a $N=36$ knot surface (Case \#6 vs. Case \#5); the DIC residual levels drop to $1.8 \%$ of the dynamic range. 
Based on the comparison of Cases \#1 and \#5 on the one hand, and \#5 and \#6 on the other hand, the BCCs and the NURBS surface parameterization both play a role in improving the registration quality through decreased residuals. In the present case, the impact of $\mathrm{BBC}$ is of lesser importance compared to the NURBS parameterization when the displacement and strain values are compared. Concerning the NURBS parametrization, the root mean square (RMS) of the difference between the displacement fields (resp. strain fields) obtained with Case \#1 and Case \#5 indicates a gap of $4 \%$ (resp. $5 \%$ ). With respect to BCCs, a similar though smaller trend is observed, namely the RMS of the difference between displacement (resp. strain) fields determined in Cases \#5 and \#6 is equal to $0.6 \%$ (resp. $0.5 \%$ ). While the BCCs are helpful in partially explaining the changes of residuals with the torsional loading, the change in lighting conditions is not detrimental to the estimation of the kinematic fields. Nonetheless it has to be emphasized that the BCCs provide a better registration quality (i.e., lower residuals). The residual maps after BCCs highlight only the remaining interpolation errors and sensor noise. They could be further exploited to detect finer singularities such as microcracks if they were present. 


\section{Conclusions}

In this study the importance of including brightness and contrast corrections (BCCs) that occur when twist is applied is investigated. The introduction of BCCs with a set of polynomial functions in the global stereocorrelation formalism is presented. The role of the number of knots used in defining the ROI and the degree of the polynomials used for the NURBS surface are also investigated. The results suggest that it is necessary to leave a certain degree of freedom to the NURBS surface in order to address torque-related sources of error in stereocorrelation formalisms based on free-form surface definitions. When the surface definitions are overly constrained (i.e., by including only a few knots or by using polynomials of low degree), the registration results may be over-regularized. The BCC lowers the final residuals.

\section{Acknowledgements}

This material is based, in part, upon work supported by the Air Force Office of Scientific Research (AFOSR) under award number FA9550-16-1-0438.

\section{References}

[1] A. Charbal, J.-E. Dufour, A. Guery, F. Hild, S. Roux, L. Vincent, and M. Poncelet, "Integrated Digital Image Correlation considering gray level and blur variations: Application to distortion measurements of IR camera," Optics and Lasers in Engineering, vol. 78, pp. 75-85, 2016.

[2] B. Beaubier, J. E. Dufour, F. Hild, S. Roux, S. Lavernhe, and K. LavernheTaillard, "CAD-based calibration and shape measurement with stereo-DIC," Experimental Mechanics, Volume 54, Issue 3, p 329-341, 2014.

[3] J. E. Dufour, B. Beaubier, F. Hild, and S. Roux, "CAD-based Displacement Measurements with Stereo-DIC,' Experimental Mechanics, Volume 55, p 1657-1668, 2015.

[4] L., Piegl and W., Tiller “The NURBS Book," Monographs in Visual Communication, Springer, 2nd edition. 
[5] ASTM E8/E8M - 11 "Standard Test Methods for Tension Testing of Metallic Materials," ASTM International. 